\title{
Efeito da borda na estrutura e estoque de carbono de uma Floresta Estacional Semidecidual
}

\author{
Samuel José Silva Soares Da Rocha ${ }^{1}$ Carlos Moreira Miquelino Eleto Torres ${ }^{1}$ Laércio Antônio Gonçalves \\ Jacovine $^{1}$ Bruno Leão Said Schettini ${ }^{1}$ Paulo Henrique Villanova ${ }^{1}$ Maria Paula Miranda Xavier Rufino ${ }^{1}$ \\ Águida Beatriz Traváglia Viana ${ }^{1}$ \\ ${ }^{1}$ Universidade Federal de Viçosa, Avenida Peter Henry Rolfs, s/n - Campus Universitário, Viçosa - MG, 36570-977
}

*Author for correspondence: blsschettini@gmail.com

Received: December 2018 / Accepted: February 2019 / Published: June 2019

\section{Resumo}

A influência do gradiente ambiental na estrutura e no estoque de carbono pode auxiliar na compreensão da estocagem de carbono na Mata Atlântica e na tomada de decisão sobre metodologias e espécies a serem usadas na restauração ecológica do bioma nos diferentes gradientes. A estrutura e o estoque de carbono em duas porções do terreno, borda e interior, foram avaliados. A ordenação das comunidades foi verificada pela análise de escalonamento multidimensional não métrica (NMDS) e a análise de espécies indicadoras (ISA) foi realizada. O efeito de borda influencia na diferenciação da composição florística e estrutura entre os dois estratos e provocou diferença significativa ANOVA (p-valor $=0,002$ ) a $5 \%$ de significância no estoque de carbono. Na região da borda foi encontrada uma estimativa de $45,43 \mathrm{MgC}^{-1}$ e no interior $63,71 \mathrm{MgC} \mathrm{ha} \mathrm{ha}^{-1}$. As espécies Aparisthmium cordatum (A.Juss.) Baill. e Cupania vernalis Cambess. são indicadoras do ambiente de borda no fragmento. Já na região do interior as espécies Apuleia leiocarpa (Vogel) J.F.Macbr., Coussarea verticillata Müll.Arg., Dalbergia nigra (Vell.) Allemao ex Benth e Melanoxylon brauna Schott foram significativas pelo teste de Monte Carlo. Conclui-se que o efeito de borda afeta a estrutura, diversidade e estoque de carbono no fragmento florestal.

Palavras-chave: Espécies Indicadoras,

Fitossociologia, Gradiente Ambiental.

\section{Abstract}

The influence of the environmental gradient on the structure and the carbon stock can help in understanding the carbon storage in the Atlantic Forest and in the decision making about methodologies and species to be used in the ecological restoration of the biome in the different gradients. For this, the structure and the carbon stock in two portions of the terrain, edge and inside, were evaluated. The ordering of the communities was verified by non - metric multidimensional scaling analysis (MMSA) and indicator species analysis (ISA) was performed. The edge effect influences the differentiation of floristic composition and structure between the two strata and caused a significant difference $(\mathrm{p}$-value $=0.002)$ at $5 \%$ significance in the carbon stock. In the region of the edge, an estimate of $45.43 \mathrm{MgC} \mathrm{ha}^{-1}$ was found and at the top of the hill was $63.71 \mathrm{MgC} \mathrm{ha}{ }^{-1}$. The species Aparisthmium cordatum (A.Juss.) Baill. and Cupania vernalis Cambess. are indicative of the edge environment in the fragment. In the inside the species Apuleia leiocarpa (Vogel) J.F.Macbr., Coussarea verticillata Müll.Arg., Dalbergia nigra (Vell.) Allemao ex Benth and Melanoxylon brauna Schott were significant by the Monte Carlo test. It is concluded that the edge effect affects the structure, diversity and carbon stock in the seasonal semideciduous forest fracturing.

Keywords: Environmental Gradient, Indicators Species, Phytosociology.

\section{Introdução}

A perda e a fragmentação do habitat, resultantes principalmente do aumento dos impactos humanos relacionados ao uso da terra, representam ameaças consideráveis para a biodiversidade (Ren et al., 2017; Sousa et al., 2017; Xie et al. 2017). No Brasil, a Mata Atlântica é uma das florestas tropicais que mais foram afetadas por esse processo (Scarano; Ceotto, 2015). As estimativas disponíveis na literatura de cobertura vegetal do bioma, variam entre 11-16\%, porém, Rezende et al. (2018) observaram 32 milhões de ha de vegetação nativa, o que representa $28 \%$.

No processo de fragmentação ambiental, o efeito de borda é um dos maiores riscos para manutenção da biodiversidade (BRASIL et al., 2013). Nesse ambiente, avaliar a influência do efeito de borda na estrutura e consequentemente no estoque de carbono, pode auxiliar na compreensão do bioma e em processos de tomada de decisão que visem à restauração ecológica. Poucos estudos relatam a importância relativa dos atributos florestais e fatores ambientais para o funcionamento do ecossistema (Poorter et al., 2015).

Uma vez que muitas mudanças visíveis da composição e da riqueza da comunidade, bem como nas condições climáticas, estão relacionadas a diferenças ambientais (BRAMBACH et al., 2017), assim os efeitos da borda de fragmentos florestais são importantes fontes de estudos (KRÖMER et al., 2013; XU et al., 2017).

Além disso, a detecção de espécies que melhor caracterizam esses gradientes é um passo importante na avaliação de classificações na ecologia comunitária (Siddig et al., 2016) e podem servir como indicadores que balizem a restauração ecológica no bioma da Mata Atlântica.

A vegetação reflete mudanças no ambiente e a análise de espécies indicadoras (ISA) ilustra como essa mudanças revelam padrões na composição da vegetação em relação aos gradientes ambientais (Vilches et al., 2013; Carvalho et al., 2017). Esse índice desenvolvido por Dufrêne; Legendre (1997) é uma metodologia para identificar espécies indicadoras, assumindo que, para que uma espécie seja um indicador apropriado de uma determinada condição (por exemplo, um tipo de habitat, estado ambiental ou um tipo de comunidade) (Mota et al., 2014).

Os tipos de associações ecológicas nos estratos podem ser distintos com base nos valores dos indicadores. Desse modo objetivou-se avaliar a estrutura, diversidade e 
estoque de carbono na borda e no interior de um fragmento de Floresta Estacional Semidecidual em Viçosa, MG. A influência do gradiente ambiental na estrutura e no estoque de carbono pode auxiliar na compreensão da estocagem de carbono na Mata Atlântica e na tomada de decisão sobre metodologias e espécies a serem usadas na restauração ecológica do bioma nos diferentes gradientes. Desse modo objetivou-se avaliar a estrutura, diversidade e estoque de carbono na borda e no interior de um fragmento de Floresta Estacional Semidecidual em Viçosa, MG.

\section{Material e métodos}

Descrição da área de estudo

A coleta de dados foi realizada em um fragmento florestal de 14,8 ha em área de Floresta Estacional Semidecidual Montana (IBGE, 2012) localizada no município de Viçosa, MG, com coordenadas geográficas $20^{\circ} 46^{\prime} 16,95^{\prime \prime} \mathrm{S}$ e $42^{\circ} 52^{\prime} 11,16^{\prime \prime} \mathrm{O}$.

O clima local é do tipo Cwa, conforme classificação de Köppen. A média da temperatura, umidade e precipitação anual para o período de 1968 a 2015 é de $21,9^{\circ} \mathrm{C}, 79 \%$ e $1.274 \mathrm{~mm}$, respectivamente (UFV, 2016). A região de Viçosa possui gradientes pedogeomorfológico em que os topos de morros são tomados por Latossolos Distróficos ricos em alumínio, as rampas coluviais por Latossolos mais rasos e câmbicos e os fundos das grotas apresentam-se com o predomínio de Cambissolos Epieutróficos ricos em nutrientes (Ferreira Júnior et al., 2012).

\section{Coleta e análise de dados}

No fragmento avaliado, foram estabelecidas sistematicamente 50 unidades amostrais contíguas, de 10 x $10 \mathrm{~m}(0,01 \mathrm{ha})$. As parcelas foram divididas $50 \%$ alocadas na borda $(0-50 \mathrm{~m})$ e os outros $50 \%$ no interior da floresta $(50-100 \mathrm{~m})$

Todos os indivíduos com dap $\geq 5 \mathrm{~cm}$ foram mensurados e identificados botanicamente. Para a análise da diversidade florística foram utilizados o Índice de diversidade de Shannon-Weaver (H') e Equabilidade de Pielou (J).

O índice de diversidade de Shanon-Weaver foi calculado utilizando a equação 1 :

$$
H^{\prime}=\frac{\left[N^{*} \ln (N)-\sum_{i=1}^{s} n_{i} * \ln \left(n_{i}\right)\right]}{N}
$$

Em que:

$\mathrm{N}$ = número total de indivíduos ou fustes amostrados; $\mathrm{n}_{\mathrm{i}}=$ número de indivíduos ou fustes amostrados da i-ésima espécie; e

$\ln =$ logaritmo neperiano.

A equabilidade de Pielou (J) foi calculada utilizando a equação 2:

$$
J=\frac{H^{\prime}}{H_{\max }}
$$

Em que:

$\mathrm{H}_{\max }=\ln (\mathrm{S})$

$\mathrm{S}=$ número total de espécies amostradas; e

H' = índice de diversidade de Shannon-Weaver.
Para a determinação das espécies indicadoras de cada uma das porções do terreno, foi realizada uma análise de espécies indicadoras (ISA) (Dufrêne e Legendre, 1997). As análises foram realizadas no programa $\mathrm{R}(\mathrm{R}$ DEVELOPMENT CORE TEAM, 2012). A significância dos valores indicadores foi avaliada pelo teste de permutação de Monte Carlo.

A ordenação das comunidades foi verificada pela análise de escalonamento multidimensional não métrica (NMDS). O coeficiente de similaridade de Bray-Curtis foi aplicado para a matriz de similaridade.

A biomassa e o estoque de carbono foram calculados com base nas equações 3 e 4, propostas por Amaro (2010) para a uma Floresta Estacional Semidecidual em Viçosa, MG, apresentadas a seguir:

$$
\begin{gathered}
\mathrm{BF}_{\mathrm{cc}}=0,024530 \times \mathrm{DAP}^{2,443356} \times \mathrm{Ht}^{0,423602} \\
\overline{\mathrm{R}}^{2}=95,01 \%
\end{gathered}
$$

Em que:

$\mathrm{BF}_{\mathrm{cc}}$ - Biomassa do fuste com casca, $\mathrm{Mg} \mathrm{ha}^{-1}$;

DAP- Diâmetro a 1,30 m de altura do solo, em cm;

Ht- Altura total, em m;

$$
\begin{gathered}
\mathrm{CF}_{\mathrm{cc}}=0,0130045 \times \mathrm{DAP}^{2,484657} \times \mathrm{Ht}^{0,426965} \\
(\text { Equação 4) } \\
\overline{\mathrm{R}}^{2}=94,74 \%
\end{gathered}
$$

Em que:

$\mathrm{CF}_{\mathrm{cc}^{-}}$- Carbono do fuste com casca, $\mathrm{Mg} \mathrm{ha}^{-1}$

DAP- Diâmetro a 1,30 m de altura do solo, em cm;

Ht- Altura total, em m.

\section{Resultados}

Estrutura e diversidade florística

Foram encontradas 97 espécies distribuídas, 86

\begin{tabular}{|c|c|c|}
\hline Parâmetro & Terço médio & Terço superior \\
\hline $\mathrm{Da}\left(\right.$ ind ha $\left.^{-1}\right)$ & 1376 & 1348 \\
\hline Riqueza & 80 & 67 \\
\hline Famlías & 38 & 32 \\
\hline $\mathrm{B}\left(\mathrm{m}^{2} \mathrm{ha}^{-1}\right)$ & 5,45 & 6,57 \\
\hline $\mathrm{H}^{\prime}$ & 3,81 & 3,53 \\
\hline $\mathrm{J}$ & 0,87 & 0,84 \\
\hline
\end{tabular}
gêneros em 41 famílias botânicas. A diversidade (H') e a equabilidade ( $\mathrm{J}$ ) na borda foram de 3,6 e 0,86 , respectivamente. Para o interior do fragmento foi de 3,53 e 0,84 , respectivamente.

Tabela 1 - Estimativas de parâmetros populacionais do fragmento florestal amostrados no município de Viçosa, Minas Gerais

Em que: $\mathrm{Da}=$ número de indivíduos por unidade de área; $\mathrm{B}=$ área basal em $\mathrm{m}^{2} \mathrm{ha}^{-1} ; \mathrm{H}^{\prime}$ = Índice de diversidade de Shannon e J = Equabilidade de Pielou.

O efeito de borda influencia na diferenciação da composição florística entre os dois estratos e esta pode ser observada na figura a seguir, pela ordenação das parcelas de estudo (Figura 1). 


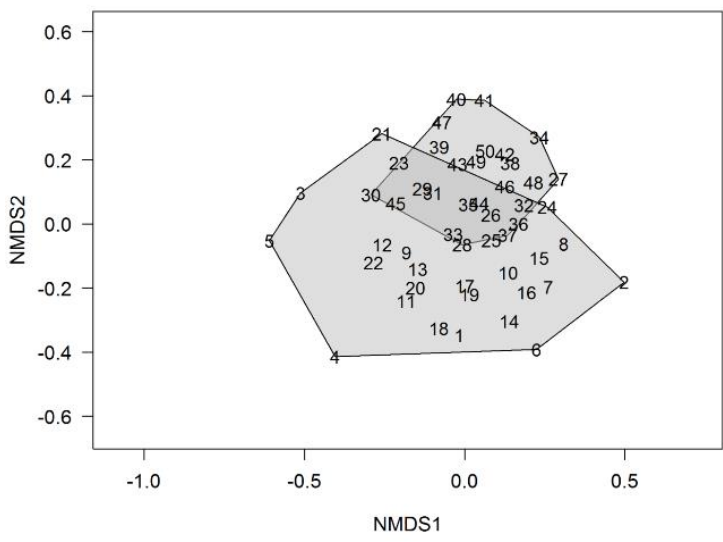

Figura 1 - Ordenação das parcelas em uma Floresta Estacional Semidecidual, Viçosa, MG.

$\mathrm{Na}$ borda, as espécies com maior VI foram Myrcia splendens $(6,83 \%)$, Siparuna guianensis $(5,76 \%)$, Apuleia leiocarpa $(5,67 \%)$ e Garcinia gardneriana $(5,15 \%)$. No interior, as espécies com maior VI foram Apuleia leiocarpa (15,24\%), Coussarea verticillata (5,77\%), Prunus myrtifolia (5,64\%), Myrcia splendens $(5,51)$ (Tabela 2).

Tabela 2 - Estrutura da comunidade na borda e no interior de um fragmento de Floresta Estacional Semidecidual em Viçosa, MG

\begin{tabular}{|c|c|c|c|c|c|c|c|c|}
\hline \multirow{2}{*}{ Nome Científico } & \multicolumn{4}{|c|}{ Borda } & \multicolumn{4}{|c|}{ Interior } \\
\hline & $\overline{\mathrm{DR}}$ & FR & DoR & VI & DR & FR & DoR & VI \\
\hline Myrcia splendens (Sw.) DC. & 7,9 & 7,0 & 5,6 & 6,8 & 7,4 & 5,4 & 3,8 & 5,5 \\
\hline Siparuna guianensis Aubl. & 9,3 & 5,9 & 2,1 & 5,8 & 8,3 & 5,4 & 1,6 & 5,1 \\
\hline Apuleia leiocarpa (Vogel) J.F.Macbr. & 3,8 & 2,7 & 10,5 & 5,7 & 11,3 & 7,8 & 26,6 & 15,2 \\
\hline Garcinia gardneriana (Planch. \& Triana) Zappi & 8,1 & 4,7 & 2,6 & 5,2 & 2,4 & 2,9 & 0,8 & 2,0 \\
\hline Jacaranda macrantha Cham. & 4,4 & 5,1 & 3,0 & 4,1 & 3,9 & 4,5 & 1,0 & 3,1 \\
\hline Prunus myrtifolia (L.) Urb. & 2,6 & 3,1 & 5,1 & 3,6 & 5,9 & 5,4 & 5,6 & 5,6 \\
\hline Cupania vernalis Cambess. & 3,2 & 2,7 & 4,5 & 3,5 & 0,6 & 0,8 & 0,7 & 0,7 \\
\hline Bathysa nicholsonii K.Schum. & 3,5 & 3,9 & 2,0 & 3,1 & 0,9 & 1,2 & 0,4 & 0,9 \\
\hline Attalea dubia (Mart.) Burret & 1,2 & 1,6 & 5,8 & 2,8 & 0,9 & 1,2 & 3,8 & 2,0 \\
\hline Machaerium brasiliense Vogel & 2,3 & 2,7 & 3,4 & 2,8 & 0,3 & 0,4 & 1,0 & 0,6 \\
\hline Protium warmingianum Marchand & 3,5 & 1,6 & 3,4 & 2,8 & 1,5 & 1,7 & 1,8 & 1,7 \\
\hline Mabea fistulifera Mart. & 3,2 & 3,5 & 1,7 & 2,8 & 1,2 & 1,7 & 0,6 & 1,2 \\
\hline Casearia ulmifolia Vahl ex Vent. & 2,3 & 3,1 & 2,5 & 2,7 & 5,0 & 5,4 & 3,9 & 4,8 \\
\hline Cupania tenuivalvis Radlk. & 2,0 & 2,0 & 3,3 & 2,4 & 2,4 & 2,5 & 2,1 & 2,3 \\
\hline Eriotheca candolleana (K.Schum.) A.Rol & 1,7 & 2,3 & 1,8 & 2,0 & 0,3 & 0,4 & 0,2 & 0,3 \\
\hline Copaifera langsdorffii Desf. & 1,2 & 1,6 & 2,8 & 1,9 & 0,6 & 0,8 & 3,0 & 1,5 \\
\hline Ocotea dispersa (Nees \& Mart.) Mez & 1,5 & 2,0 & 2,1 & 1,8 & 3,6 & 3,3 & 1,1 & 2,7 \\
\hline Annona cacans Warm. & 1,2 & 1,2 & 3,1 & 1,8 & 3,3 & 4,1 & 7,1 & 4,8 \\
\hline Aparisthmium cordatum (A.Juss.) Baill & 2,0 & 2,3 & 0,8 & 1,7 & - & - & - & - \\
\hline Cordia sellowiana Cham. & 1,5 & 2,0 & 1,1 & 1,5 & 0,3 & 0,4 & 0,1 & 0,3 \\
\hline Qualea jundiahy Warm. & 0,3 & 0,4 & 3,6 & 1,4 & 0,6 & 0,8 & 0,1 & 0,5 \\
\hline Ocotea odorifera (Vell.) Rohwer & 1,2 & 1,2 & 1,9 & 1,4 & 0,3 & 0,4 & 0,1 & 0,3 \\
\hline Annona sylvatica A.St.-Hil. & 1,7 & 1,6 & 0,9 & 1,4 & 0,6 & 0,8 & 0,2 & 0,6 \\
\hline Coutarea hexandra (Jacq.) K.Schun & 1,2 & 1,2 & 1,6 & 1,3 & 1,2 & 1,2 & 0,4 & 0,9 \\
\hline Piptadenia gonoacantha (Mart.) Macbr & 1,2 & 0,8 & 1,7 & 1,2 & - & - & - & - \\
\hline Coussarea verticillata Müll.Arg. & 1,2 & 0,8 & 1,6 & 1,2 & 6,5 & 5,8 & 5,0 & 5,8 \\
\hline Amaioua guianensis Aubl. & 1,2 & 1,6 & 0,7 & 1,1 & 2,1 & 2,9 & 1,5 & 2,2 \\
\hline Matayba elaeagnoides Radlk. & 0,6 & 0,4 & 2,4 & 1,1 & 0,3 & 0,4 & 0,1 & 0,3 \\
\hline Demais espéc & 25,3 & 31,3 & 18,5 & 25,0 & 28,5 & 32,5 & 27,4 & 29,5 \\
\hline Tota & 100 & 100 & 100 & 100 & 100 & 100 & 100 & 100 \\
\hline
\end{tabular}

Análise de espécies indicadoras

Do total de espécies amostradas, duas apresentaram preferência pela área borda do fragmento avaliado (Tabela 3).
Tabela 3 - Espécies indicadoras da borda e do interior de um fragmento de Floresta Estacional Semidecidual, Viçosa, MG

\begin{tabular}{cccc} 
Limite & Espécies & Valor indicador & $\mathrm{p}$-valor \\
\hline \multirow{3}{*}{ Borda } & Aparisthmium cordatum & 0,490 & $0,015^{*}$ \\
& Cupania vernalis & 0,487 & $0,050^{*}$ \\
\hline \multirow{3}{*}{ Interior } & Apuleia leiocarpa & 0,730 & $0,005^{* *}$ \\
& Coussarea verticillata & 0,655 & $0,010^{* *}$ \\
& Dalbergia nigra & 0,447 & $0,040^{*}$ \\
& Melanoxylon brauna & 0,447 & $0,050^{*}$ \\
\hline
\end{tabular}

* Significativo ao nível de 5\% ** Significativo ao nível de $1 \%$

Já para a área do interior, quatro espécies apresentam fidelidade, ou seja, significância estatística (p-valor>0,05) pelo teste de permutação de Monte Carlo.

\section{Estoque de carbono}

Observou-se que o efeito de borda provocou diferença significativa ANOVA (p-valor < 0,05) a $5 \%$ de significância na biomassa e estoque de carbono. Na região da borda foi encontrada uma estimativa de $45,43 \mathrm{Mg} \mathrm{ha}^{-1}$ e no interior de 63,71 $\mathrm{Mg} \mathrm{ha}^{-1}$ (Figura 2).
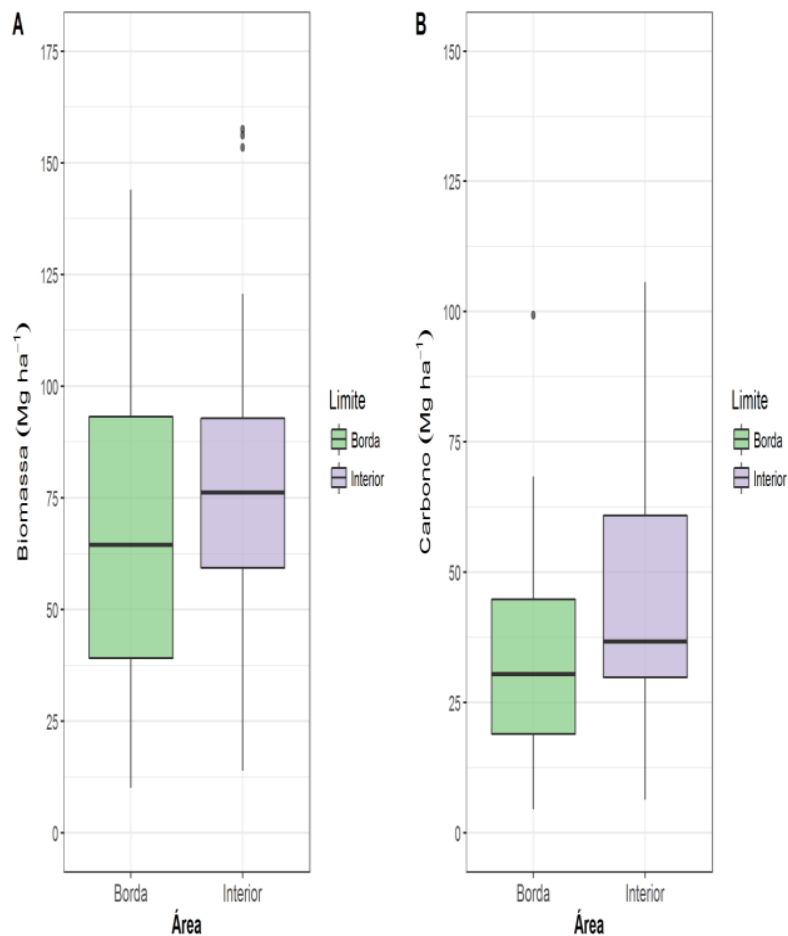

Figura 2 - Acúmulo de biomassa (A) e estoque de carbono (B) em dois estratos (borda e interior) de um fragmento de Floresta Estacional Semidecidual, Viçosa, MG

\section{Discussão}

De modo geral, os estratos na borda e no interior apresentam alta diversidade, os valores do índice de diversidade de Shannon e Equabilidade de Pielou foram de $\mathrm{H}^{\prime}=3,81$ e 3,53 e $\mathrm{J}=0,87$ e 0,84 respectivamente, e é considerado alto se comparado aos observados na literatura em formações semelhantes (Brito; Carvalho, 
2014). Contudo, a estrutura e diversidade florística foi influenciado pelas condições do local, houve redução da densidade e riqueza com o efeito da borda do fragmento. O padrão de distribuição e estrutura pode ter sido resultado das interações de fatores ambientais. As florestas são espacialmente heterogêneas devido a uma combinação complexa de condições ambientais, topográficas e perturbação humana (Corona-núñez et al. 2017).

A análise de espécies indicadoras reforçou a existência de diferenças florístico-estruturais entre os estratos, apontando espécies com associações em cada área. A classificação, a distribuição e o reconhecimento de espécies indicadoras são afetados por várias variáveis ambientais, como as condições do solo, perturbação, microclima e as práticas agrícolas prevalecentes (Iqbal et al., 2018). As espécies Aparisthmium cordatum e Cupania vernalis são indicadoras do ambiente na borda do fragmento. Já na região do interior do fragmento as espécies Apuleia leiocarpa, Coussarea verticillata, Dalbergia nigra e Melanoxylon brauna foram significativas pelo teste de Monte Carlo. Essas espécies apresentam um alto grau de ocupação da área, alta estimativa de área basal, sendo, portanto, indivíduos de alto porte e adultos.

Os valores de estoque de carbono observados foram próximos aos encontrados na literatura para formações semelhantes de Floresta Estacional Semidecidual (Souza et al., 2012; Torres et al., 2013; Gaspar et al., 2014). A alteração na estrutura e composição florística da comunidade resulta em um decréscimo do estoque de carbono. Apesar de estudos apontarem uma relação positiva entre a diversidade florestal e a biomassa acima do solo (Con et al., 2013; Potter; Woodall, 2014; Ali; Yan, 2017) a presença de indivíduos de maior porte no interior, influenciaram a capacidade desse estrato em ofertar o serviço ambiental de estoque de carbono. Isso pode ser reflexo da menor perturbação dessas áreas, o que garantiu a permanência dos maiores indivíduos, que contribuem desproporcionalmente para o maior aporte de carbono (Poorter et al., 2015).

Assim, o estoque de carbono do ecossistema foi influenciado pelos efeitos de borda. Em comparação com florestas intactas, os fragmentos florestais têm uma proporção maior de habitat de borda que a outros tipos de habitat, levando a um maior potencial de efeitos de borda. $\mathrm{O}$ estoque de carbono é suscetível aos efeitos de borda, com menor estocagem nas bordas em comparação com o interior da floresta, devido aos maiores valores de $\mathrm{Ht}$ e DAP das árvores nesses locais na ocasião. As bordas da floresta podem ser vistas como zonas de amortecimento através das quais as condições ambientais mudam progressivamente com a distância, levando a impactos significativos sobre a biomassa acima do solo (Barros; Fearnside, 2016)

Além disso, a fragmentação pode afetar a densidade de carbono do ecossistema, por meio do aumento das áreas de borda e consequente mudança na composição de espécies. Essas mudanças, por sua vez, embora indiretamente, afetam as funções e processos dos ecossistemas, como a produtividade primária, os reservatórios de $\mathrm{C}$ acima e abaixo do solo e a dinâmica de carbono (Ziter et al., 2013).

Por fim, nota-se que os resultados gerados podem balizar programas de restauração e/ou manejo de terras, com a recomendação de espécies que tenham condições adequadas para seu desenvolvimento de acordo com as características do local. O entendimento dos efeitos da fragmentação na composição e estrutura dos fragmentos informações podem auxiliar no planejamento de políticas para gerenciar a biodiversidade tropical (Sullivan et al., 2017), sobretudo no domínio da Mata Atlântica.

\section{Conclusões}

Conclui-se que o efeito de borda afeta a estrutura, diversidade e estoque de carbono no fragmento de Floresta Estacional Semidecidual. O interior da floresta apresenta maior estoque de carbono na área. As espécies indicadoras de cada porção do terreno podem ser utilizadas para compreender influência do gradiente ambiental na oferta de serviços ambientais do ecossistema, tal como o estoque de carbono.

\section{Referências Bibliográficas}

Ali A, Mattsson E (2017) Disentangling the effects of species diversity, and intraspecific and interspecific tree size variation on aboveground biomass in dry zone homegarden agroforestry systems. Science of The Total Environment, $598 \quad$ (C): 38-48. doi: 10.1016/j.scitotenv.2017.04.131

Ali A, Yan ER (2017) The forest strata-dependent relationship between biodiversity and aboveground biomass within a subtropical forest. Forest Ecology and Management, $401 \quad$ (C): $125-134 . \quad$ doi: 10.1016/j.foreco.2017.06.056

Amaro MA (2010) Quantificação do estoque volumétrico, de biomassa e de carbono em uma Floresta Estacional Semidecidual no Município de Viçosa-MG. Tese, Departamento de Engenharia Florestal, Universidade Federal de Viçosa, $168 \mathrm{f}$.

Bangroo SA, Najar GR, Rasool A (2017) Effect of altitude and aspect on soil organic carbon and nitrogen stocks in the Himalayan Mawer Forest Range. Catena, 158 (C): 6368. doi: 10.1016/j.catena.2017.06.017.

Barros HS, Fearnside PM (2016) Soil Carbon Stock Changes Due to Edge Effects in Central Amazon Forest Fragments. Forest Ecology and Management, 379(1): 3036. https://doi.org/10.1016/j.foreco.2016.08.002

Brambach F, Leuschner C, Tjoa A, Culmsee H (2017) Diversity, endemism, and composition of tropical mountain forest communities in Sulawesi, Indonesia, in relation to elevation and soil properties. Perspectives in Plant Ecology, Evolution and Systematics, 27 (C): 68-79. doi: 10.1016/j.ppees.2017.06.003

Brasil LS, Giehl NFS, Santos JO, Santos AO, Marimom BS, Junior BHM (2013) Efeito de borda sobre a camada de serapilheira em área de cerradão no leste de Mato Grosso. Biotemas, 26 (3): 37-47. doi: 10.5007/21757925.2013v26n3p37

Brito OS, Carvalho FA (2014) Estrutura e diversidade arbórea da Floresta Estacional Semidecidual secundária no Jardim Botânico da Universidade Federal de Juiz de Fora. Rodriguésia - Instituto de Pesquisas Jardim Botânico do Rio de Janeiro, 65 (4): 817-830. doi: 10.1590/21757860201465402

Carvalho MA, Lana CC, Bengtson P, Sá NP (2017) Late Aptian (Cretaceous) climate changes in northeastern Brazil: A reconstruction based on indicator species analysis (IndVal). Palaeogeography, Palaeoclimatology, Palaeoecology, $485 \quad$ (C): $543-560 . \quad$ doi: 10.1016/j.palaeo.2017.07.011 
Con TV, Ha DTT, Quy TH, Van Do T, Thang NT, Khiem CC, Lam VT, Sato T (2013) Relationship between aboveground biomass and measures of structure and species diversity in tropical forests of Vietnam. Forest Ecology and Management, 310 (C): 213-218. doi: 10.1016/j.foreco.2013.08.034

Corona-Núñez RO, Mendoza-Ponce A, López-Martínez R (2017) Model selection changes the spatial heterogeneity and total potential carbon in a tropical dry forest. Forest Ecology and Management, 405 (C): 69-80. doi: 10.1016/j.foreco.2017.09.018

Dufrêne M, Legendre P (1997) Species Assemblages and Indicator Species:the Need for a Flexible Asymmetrical Approach. Ecological Monographs, 67 (3): 345-366. doi: 10.1890/0012-9615(1997)067[0345:SAAIST]2.0.CO;2

Ferreira Júnior WG, Schaeffer CEGR, Silva AF (2012) Uma visão pedogeomorfológica sobre as formações florestais da Mata Atlântica. In: Martins SV (ed). Ecologia de florestas tropicais do Brasil. Viçosa: Editora UFV, p.109-142

Gaspar RO, Castro RVO, Peloso RV, Souza, FC, Martins SV (2014) Análise fitossociológica e do estoque de carbono no estrato arbóreo de um fragmento de Floresta Estacional Semidecidual. Ciência Florestal, 24 (2): 313324. doi: 10.5902/1980509814569

Instituto Brasileiro de Geografia e Estatística - IBGE (2012) Manual Técnico da Vegetação Brasileira. $2^{\mathrm{a}}$ ed. Rio de Janeiro: 275p.

Iqbal M, Khan SM, Khan MA, Ahmad Z, Ahmad H (2008) A novel approach to phytosociological classification of weeds flora of an agro-ecological system through Cluster, Two Way Cluster and Indicator Species Analyses. Ecological Indicators, 84 (C): 590-606. doi: 10.1016/j.ecolind.2017.09.023

Krömer T, Acebey A, Kluge J, Kessler M (2013) Effects of altitude and climate in determining elevational plant species richness patterns: A case study from Los Tuxtlas, Mexico. Flora - Morphology, Distribution, Functional Ecology of Plants, 208 (3): 197-210. doi: 10.1016/j.flora.2013.03.003

Mota SLL, Pereira IM, Machado EVL, Oliveira MLR, Bruzinga JS, Farnezi MMM, Júnior MSM (2014) The influence of rocky outcrops on the woody community of a cerrado stricto sensu. Floresta e Ambiente, 21 (1): 8-18. doi: 10.4322/floram.2014.009

Poorter L, Sande MTV, Thompson J, Arets EJMM, Alarcón A, Álvarez Sánchez J, Ascarrunz N, Balvanera P, Barajas-Guzmán G, Boit A, Bongers F et al (2015) Diversity enhances carbon storage in tropical forests. Global Ecology and Biogeography, 24 (11): 1314-1328. doi: 10.1111/geb.12364

Potter KM, Woodall CW (2014) Does biodiversity make a difference? Relationships between species richness, evolutionary diversity, and aboveground live tree biomass across U.S. forests. Forest Ecology and Management, Mechanisms and predictors of ecological change in managed forests: A selection of papers from the second international conference on biodiversity in forest ecosystems and landscapes, 321 (C): 117-129. doi: 10.1016/j.foreco.2013.06.026
R Development Core Team (2012). R: A Language and Environment for Statistical Computing. Vienna: $R$ foundation for Statistical Computing. http://www.Rproject.org/

Ren X, LV Y, Li M (2017) Evaluating differences in forest fragmentation and restoration between western natural forests and southeastern plantation forests in the United States. Journal of Environmental Management, 188 (C): 268-277. doi: 10.1016/j.jenvman.2016.11.068

Rezende CL, Scarano FR, Assad ED, Joly CA, Metzger JP, Strassburg BBN, Tabarelli M, Fonseca GA, Mittermeier RA (2018) From hotspot to hopespot: An opportunity for the Brazilian Atlantic Forest. Perspectives in Ecology and Conservation, 16 (4): 208 - 214. Doi: https://doi.org/10.1016/j.pecon.2018.10.002

Rodrigues LA, Carvalho DA, Filho ATO, Curi N (2007) Effects of soils and topography on the distribution of tree species in a fragment of tropical seasonal forest in Luminárias, SE Brazil. Revista Árvore, 31 (1): 25-35. doi: 10.1590/S0100-67622007000100004

Scarano FR, Ceotto P (2015) Brazilian Atlantic forest: impact, vulnerability, and adaptation to climate change. Biodiversity and Conservation, 24 (9): 2319-2331. doi: 10.1007/s10531-015-0972-y

Siddig AAH, Ellison AM, Ochs A, Villar-Leeman C, Lau MK (2016) How do ecologists select and use indicator species to monitor ecological change? Insights from 14 years of publication in Ecological Indicators. Ecological Indicators, $\quad 60: \quad 223-230 . \quad$ doi: 10.1016/j.ecolind.2015.06.036

Silva FKG, Lopes SF, Lopez LCS, Miranda de Melo JI, Trovão DMB (2014) Patterns of species richness and conservation in the Caatinga along elevational gradients in a semiarid ecosystem. Journal of Arid Environments, 110 (C): 47-52. doi: 10.1016/j.jaridenv.2014.05.011

Silva Mota G, Luz GR, Mota NM, Coutinho ES, Veloso MDM, Fernandes GW, Nunes JRF (2017) Changes in species composition, vegetation structure, and life forms along an altitudinal gradient of rupestrian grasslands in south-eastern Brazil. Flora, 238: 32-42. doi: 10.1016/j.flora.2017.03.010

Sousa TR, Costa FRC, Bentos TV, Leal Filho N, Mesquita RCG, Ribeiro IO (2017) The effect of forest fragmentation on the soil seed bank of Central Amazonia. Forest Ecology and Management, 393 (C): 105-112. doi: 10.1016/j.foreco.2017.03.020

Souza AL, Boina A, Soares CPB, Vital BR, Gaspar RO, Lana JM (2012) Phytosociological structure, volume stocks, biomass, carbon and carbon dioxide in Seasonal Semideciduos Forest. Revista Árvore, 36 (1): 169-179. doi: http://dx.doi.org/10.1590/S010067622012000100018

Sullivan MP, Talbot J, Lewis SL, Phillips OL, Qie L, Begne SK, Chave J, Cuni-Sanchez A, Hubau W, LopezGonzalez G, Miles L, Monteagudo-Mendoza A, Sonké B, Sunderland T, Ter Steege H, White LJT, Affum-Baffoe K, Aiba S, Almeida EC, Oliveira EA, Alvarez-Loayza P, Dávila EÁ, Andrade A, Aragão LEOC, Ashton P, Aymard CGA et al (2017) Diversity and carbon storage across the tropical forest biome. Scientific Reports, 7 (39102). doi: 10.1038/srep39102 
Torres CMME, Jacovine LAG, Soares CPB, Oliveira Neto SN, Santos RD, Castro Neto F (2013) Quantifying biomass and carbon stock in a seasonal semideciduous forest in Viçosa, MG, Brazil. Revista Árvore, 37 (4): 647-655. doi: 10.1590/S0100-67622013000400008

Universidade Federal de Viçosa - UFV (2016) Departamento de Engenharia Agrícola. Estação Climatológica Principal de Viçosa. Boletim meteorológico 2016. Viçosa - MG.

Vilches B, Cáceres M, Sánchez-Mata D, Gavilán RG (2013) Indicator species of broad-leaved oak forests in the eastern Iberian Peninsula. Ecological Indicators, 26 (C): 44-48. doi: 10.1016/j.ecolind.2012.10.022

Xie H, He Y, Zhang N, Lu H (2017) Spatiotemporal changes and fragmentation of forest land in Jiangxi Province, China. Journal of Forest Economics, 29 (A): 413. doi: 10.1016/j.jfe.2017.08.004

Xu M, Ma L, Jia Y, Liu M (2017) Integrating the effects of latitude and altitude on the spatial differentiation of plant community diversity in a mountainous ecosystem in
China.
PLoS
$O N E$,
12
(3). doi:

10.1371/journal.pone.0174231

Zitter C, Bennett EM, Gonzalez A (2013) Functional diversity and management mediate aboveground carbon stocks in small forest fragments. Ecosphere, 4(7): 1 - 21, 2013.

Zhang Y, Chen HYH, Taylor AR (2017) Positive species diversity and above-ground biomass relationships are ubiquitous across forest strata despite interference from overstorey trees. Functional Ecology, 31 (2): 419-426. doi: 10.1111/1365-2435.12699 